\title{
Hamiltonian Paths and Hyperbolic Patterns
}

\author{
Douglas Dunham \\ Department of Computer Science \\ University of Minnesota Duluth \\ Duluth, MN 55812-3036, USA \\ E-mail: ddunhamed.umn . edu \\ Web Site: http://www.d.umn.edu/ ddunham/
}

\begin{abstract}
In 1978 I thought it would be possible to design a computer algorithm to draw repeating hyperbolic patterns in a Poincare disk based on Hamiltonian paths in their symmetry groups. The resulting successful program was capable of reproducing each of M.C. Escher's four "Circle Limit" patterns. The program could draw a few other patterns too, but was somewhat limited. Over the years I have collaborated with students to develop algorithms that are more general and more sophisticated. I will describe these algorithms and show some of the patterns they produced.
\end{abstract}

\section{Introduction}

For more than a century mathematicians have been drawing patterns to explain concepts in hyperbolic geometry. Figure 1 shows one such pattern that appeared in a paper by the Canadian mathematician H.S.M. Coxeter [Cox57]. In 1958 Coxeter sent the Dutch artist M.C. Escher a reprint of that paper. When Escher saw

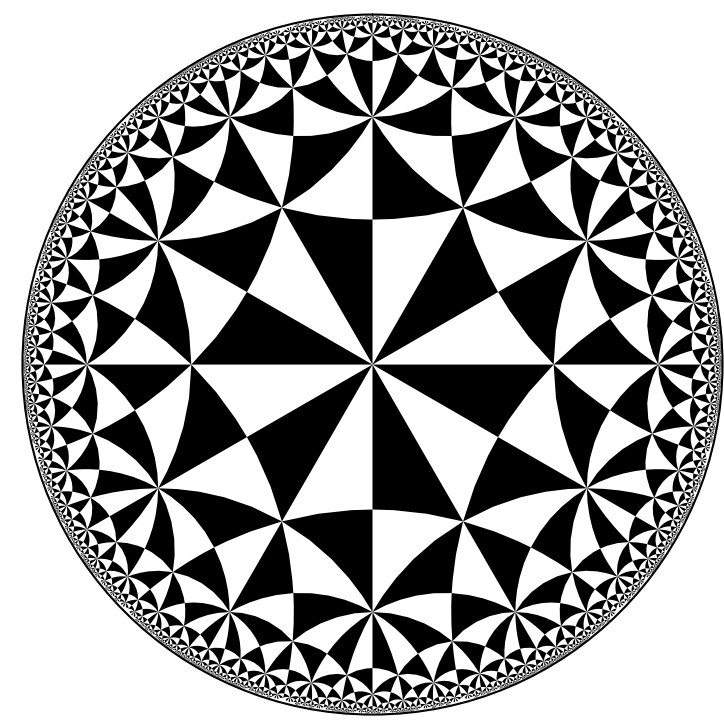

Figure 1: A hyperbolic triangle pattern.

the hyperbolic pattern, he said that it "gave me quite a shock", since it showed him how to make an infinite pattern within the confines of a finite disk. (Some of this Coxeter-Escher correspondence is recounted in [Cox79].) Later that year, with the pattern of Figure 1 as inspiration, Escher created his first artistic hyperbolic pattern, Circle Limit I, a rendition of which is shown in Figure 2 below. In my 2003 Mathematics Awareness Month essay [Dun03], I offer one explanation of how Escher might have constructed Circle Limit I from the pattern of Figure 1. Over the next two years Escher went on to create three more Circle Limit 
patterns. Since he was working by hand, for each pattern carving a woodblock with a sector of the pattern, then repeatedly rotatting and printing the woodblock to fill out the whole pattern, this was time consuming, and probably the reason he stopped at four patterns.

Around 1970 I became intrigued by Escher's mathematical art. Soon after I arrived at the University of Minnesota Duluth in 1977, I discussed the group-theoretical aspects of Escher's patterns with Joe Gallian. At that time he had undergraduate research students who were working on finding Hamiltonian cycles and paths in finite groups. Several months later it occurred to me that following a Hamiltonian path in the symmetry group of a hyperbolic pattern could lead to an algorithm to draw such patterns. Indeed, this turned out to be the case, as will be elaborated below.

In what follows, I start with a brief discussion of hyperbolic geometry, repeating patterns, and regular tessellations. Then I explain how Hamiltonian paths in hyperbolic symmetry groups were utilized in a computer program to draw repeating hyperbolic patterns. That method was the inspiration for other algorithms for drawing such patterns. Finally, I discuss possible directions of future work. Along the way I show several hyperbolic patterns.

\section{Hyperbolic Geometry, Repeating Patterns, and Regular Tessellations}

Axioms for the hyperbolic plane can be taken to be those of the Euclidean plane except that the Euclidean parallel axiom is replaced by one of its negations: given a line and a point not on it, there is more than one line through the point not meeting the original line [Gre93]. In 1901 David Hilbert proved that the entire hyperbolic plane has no smooth, isometric embedding in Euclidean 3-space [Hil01]. Thus, we must rely on Euclidean models of hyperbolic geometry in which distance is measured differently and concepts such as hyperbolic lines have interpretations as Euclidean constructs. Following Coxeter's illustration in Figure 1, Escher used the Poincaré disk model of hyperbolic geometry. In this model, hyperbolic points are just the (Euclidean) points within a Euclidean bounding circle. Hyperbolic lines are represented by circular arcs (including diameters) orthogonal to the bounding circle. For example, the backbone lines of the fish in Figure 2 lie along hyperbolic lines. Figure 3 shows that this model satisfies the hyperbolic parallel axiom.

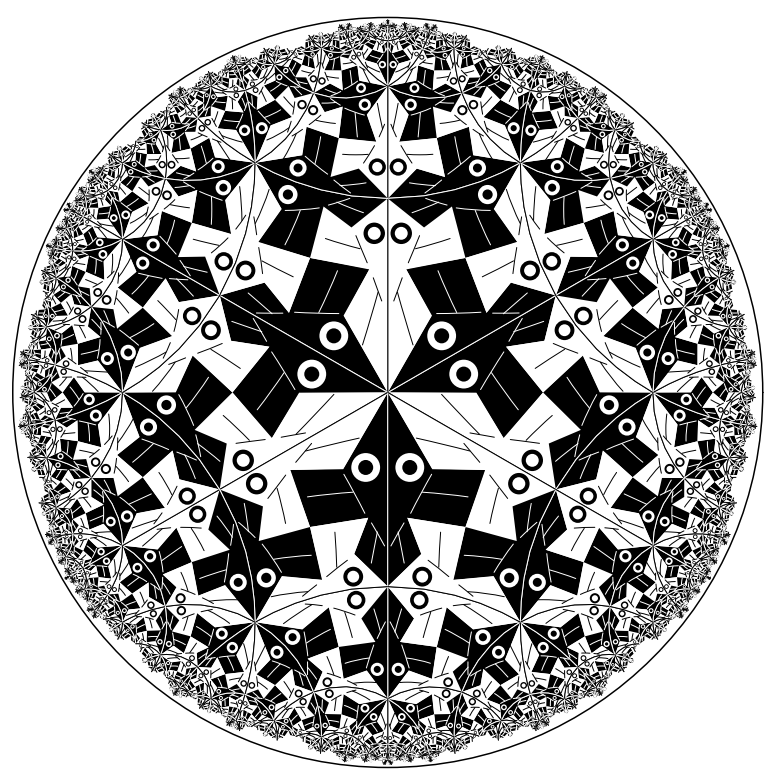

Figure 2: A rendition of Escher's Circle Limit I pattern.

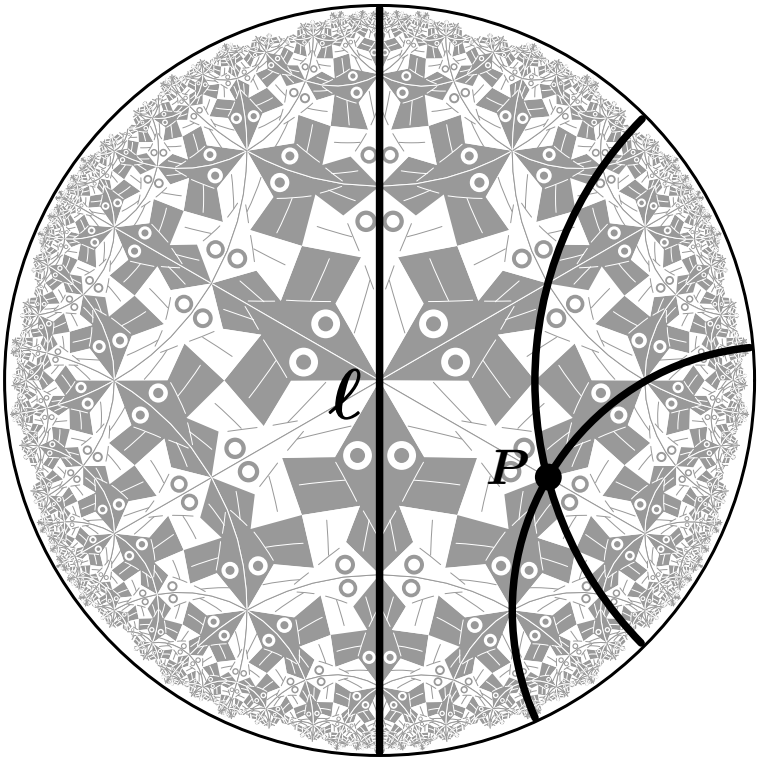

Figure 3: An example of the hyperbolic parallel property: a line $\ell$, a point $P$ not on $\ell$, and two lines through $P$ not meeting $\ell$. 
The Poincare disk model is also conformal: the hyperbolic measure of an angle is the same as its Euclidean measure. As a consequence, all the black fish in Circle Limit I have roughly the same Euclidean shape, and the same is true of the white fish. However, equal hyperbolic distances correspond to ever smaller Euclidean distances as one travels toward the edge of the disk. So all the black fish in Circle Limit I are the same (hyperbolic) size, as are all the white fish. Note that the white fish are not isometric to the black fish since the nose angle of the white fish is $90^{\circ}$ and the tail angle is $60^{\circ}$, the reverse of the nose and tail angles of the black fish. The Poincaré disk model is appealing to artists (and appealed to Escher) since an infinitely repeating pattern can be enclosed in a bounded area and shapes remain recognizable even for small copies of the motif, due to conformality. Of course Escher was more interested in the Euclidean properties of the disk model than the fact that it could be interpreted as hyperbolic geometry.

A repeating pattern in hyperbolic geometry is a regular arrangement of copies of a basic subpattern or motif. The copies should not overlap, and a characteristic of Escher's patterns is that there are also no gaps between motifs. Half of one white fish plus half of an adjacent black fish form a motif for the Circle Limit I pattern. Similarly, a white triangle and an adjacent black triangle form a motif for the pattern in Figure 1. One special repeating pattern is the regular tessellation, $\{p, q\}$, by regular $p$-sided polygons or $p$-gons, $q$ of which meet at each vertex. Figures 4 and 5 show the $\{6,4\}$ tessellation superimposed on renditions of Circle Limit I and Circle Limit IV. Similarly, Figures 6 and 7 show the $\{8,3\}$ tessellation superimposed on Circle

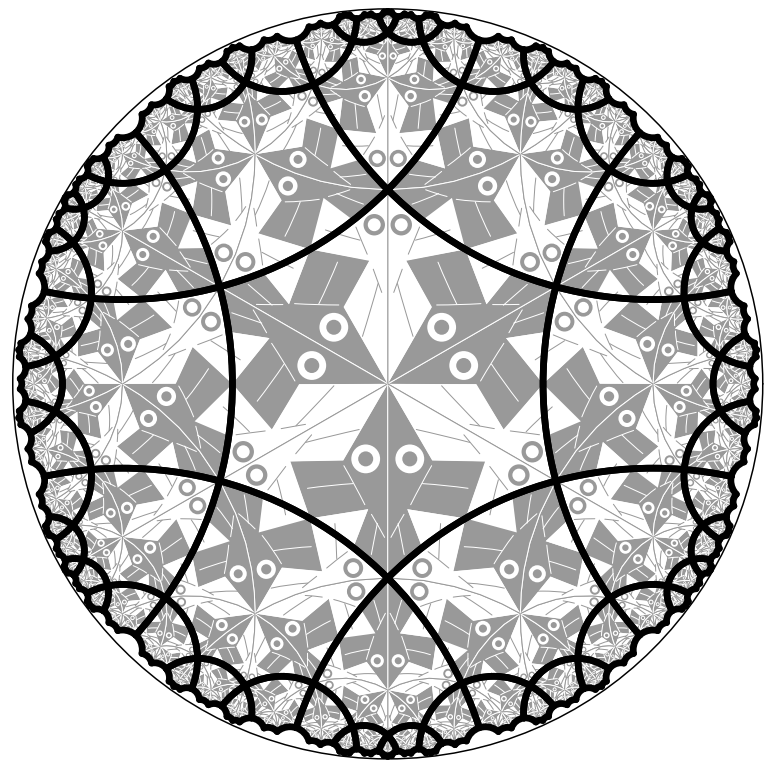

Figure 4: The $\{6,4\}$ tessellation superimposed on the Circle Limit I pattern.

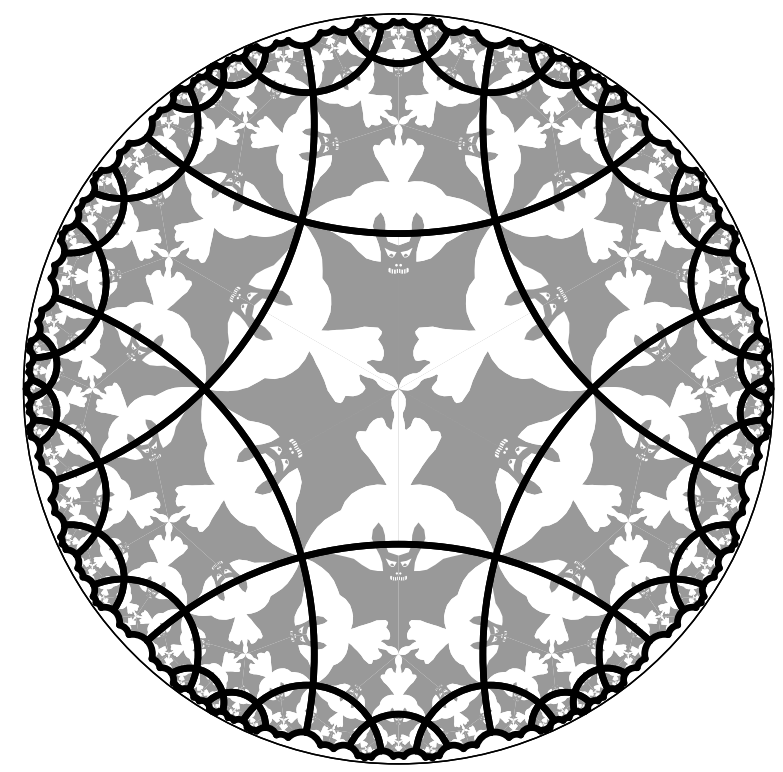

Figure 5: The $\{6,4\}$ tessellation superimposed on the Circle Limit IV pattern.

Limit II and on Circle Limit III. Note that for $\{p, q\}$ to be a tessellation of the hyperbolic plane, it is necessary that $(p-2)(q-2)>4$, otherwise one obtains one of the finitely many Euclidean or spherical tessellations. Doris Schattschneider's book Visions of Symmetry [Sch04] is the definitive reference for Escher's repeating patterns.

\section{Symmetry Groups for Hyperbolic Patterns}

A symmetry of a repeating pattern is an isometry (distance-preserving transformation) that transforms the pattern onto itself. In the Poincaré disk model, hyperbolic reflections across hyperbolic lines are inversions in the circular arcs representing those lines; reflections across diameters are ordinary Euclidean reflections. And in hyperbolic geometry, just as in Euclidean geometry, a translation is the composition of successive 


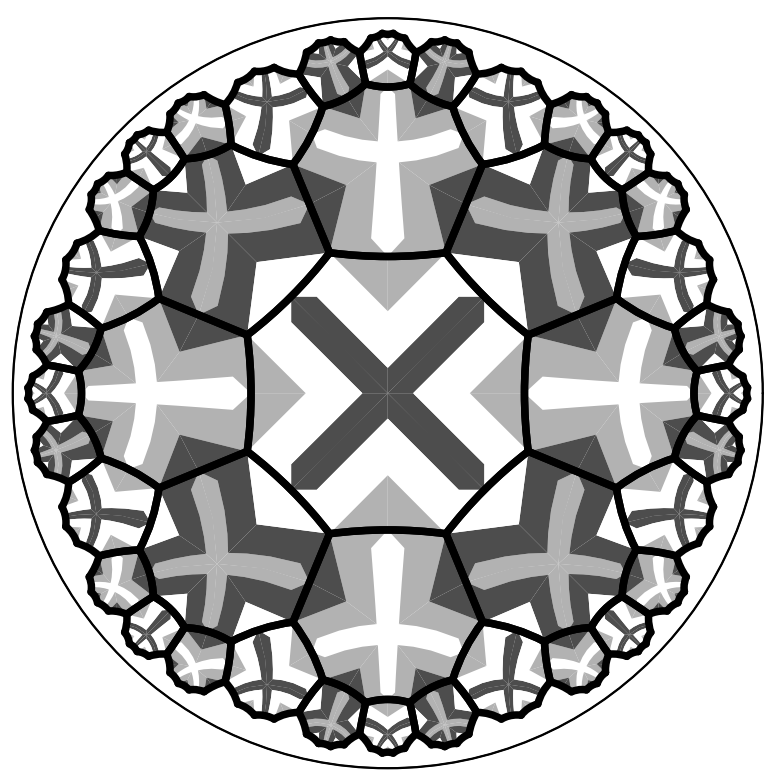

Figure 6: The $\{8,3\}$ tessellation superimposed on the Circle Limit II pattern.

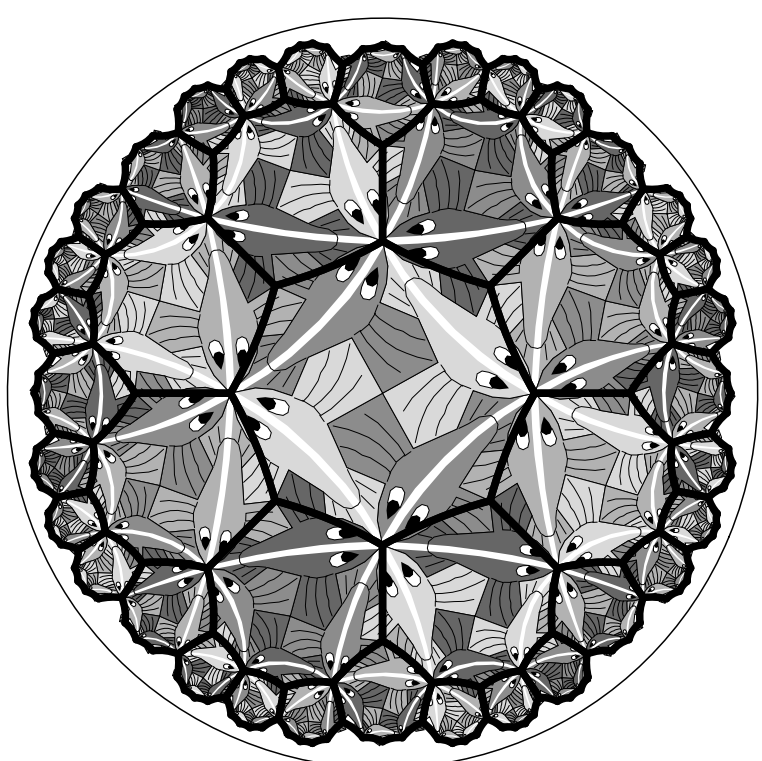

Figure 7: The $\{8,3\}$ tessellation superimposed on the Circle Limit III pattern.

reflections across two lines having a common perpendicular; the composition of reflections across two intersecting lines produces a rotation about the intersection point by twice the angle of intersection. In Circle Limit I (Figure 2), hyperbolic reflections across the backbone lines are symmetries of the repeating patttern. Other symmetries of Circle Limit I include rotations by 120 degrees about points where three black fish noses meet, rotations of 180 degrees about white fish noses and also about the points where the trailing edges of fin-tips meet, and translations by four fish-lengths along backbone lines.

The symmetry group of a pattern is the set of all symmetries of the pattern. The symmetry group of the tessellation $\{p, q\}$ is denoted $[p, q]$ and can be generated by reflections across the sides of a right triangle with acute angles of 180/p degrees and 180/q degrees. For example, [6,4] can be generated by reflections across the sides of any one of the triangles in Figure 1. The orientation-preserving subgroup of $[p, q]$ (of index 2), consists of symmetries composed of an even number of reflections, and is denoted $[p, q]^{+}$. Figure 8 shows a hyperbolic pattern with symmetry group $[5,5]^{+}$(ignoring color). This pattern uses a fish motif like that of Escher's Notebook Drawing Number 20 (p. 131 of [Sch04]) and his carved sphere with fish (p. 244 of [Sch04]); those ifsh patterns have symmetry groups $[4,4]^{+}$and $[3,3]^{+}$respectively.

There is another index 2 subgroup of $[p, q]$ that is denoted $\left[p^{+}, q\right]$ and is generated by a rotation of $360 / p$ degrees about the center of a $p$-gon and a reflection in one of its sides, where $q$ must be even so that the reflections across the sides of the $p$-gon match up. The symmetry groups of Circle Limit II and Circle Limit IV (Figure 5) is an instance of $\left[4^{+}, 6\right]$ since rotating a joined half-angel and half-devil by $90^{\circ}$ three times about their common wing tips will fill out a square; reflecting the rotated design across an edge of that square and continuing to repeat the process will produce the whole pattern (see [Dun86]). The symmetry group of Circle Limit II (Figure 6) is an instance of $\left[3^{+}, 8\right]$. Figure 9 shows a pattern of 5-armed crosses with symmetry group $\left[3^{+}, 10\right]$ that is similar to Escher's Circle Limit II. In both patterns, $120^{\circ}$ rotation centers are to the left and right of the ends of each cross arm, and $q / 2$ reflection lines pass through the center of the crosses (and the center of the bounding circle). In addition to Circle Limit IV, Escher used the group $\left[p^{+}, q\right]$ for two other "angel and devil" patterns: Notebook Drawing Number 45 and Heaven and Hell on a carved maple sphere, with symmetry groups $\left[4^{+}, 4\right]$ and $\left[3^{+}, 4\right]$ respectively (see pages 150 and 244 of [Sch04]). The angel and devil pattern is the only one Escher implemented on all three surfaces: the sphere, the Euclidean plane, and the hyperbolic plane. 


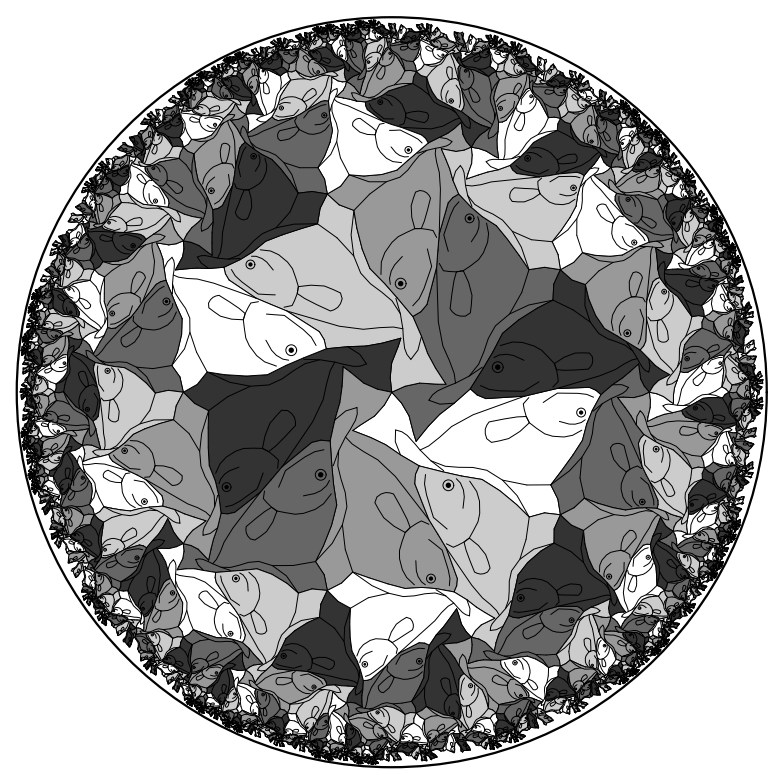

Figure 8: A hyperbolic pattern with symmetry group $[5,5]^{+}$using a fish motif like that of Escher's Notebook Drawing Number 20.

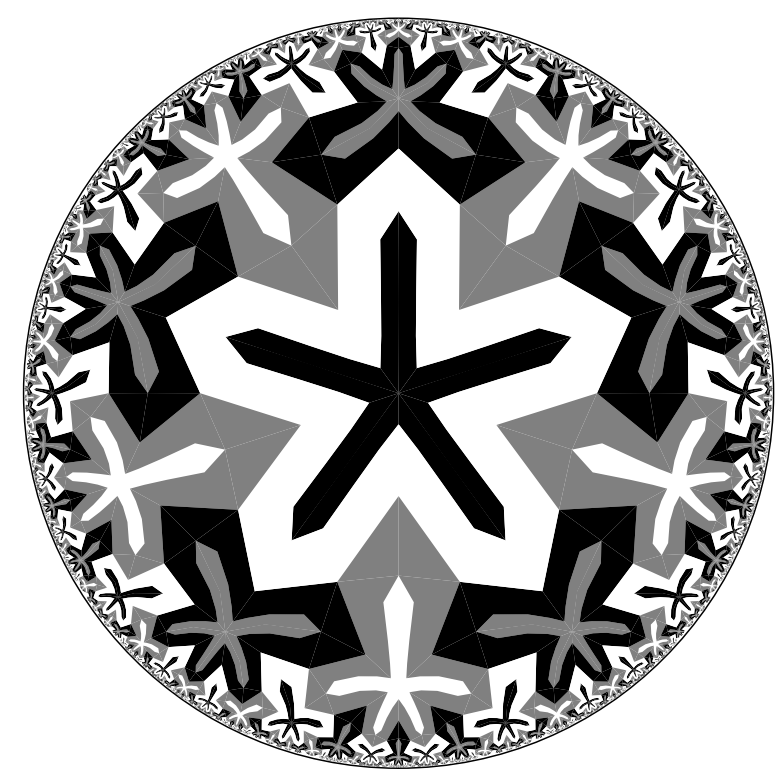

Figure 9: A hyperbolic pattern of 5-armed crosses with symmetry group $\left[3^{+}, 10\right]$.

If both $p$ and $q$ are even, there is yet another index 2 subgroup of $[p, q]$, denoted $\mathrm{cmm}_{p / 2, q / 2}$, that is generated by reflections in two adjacent sides of a rhombus with angles of $360 / p$ degrees and $360 / q$ degrees, and a 180 degree rotation about its center. This notation generalizes the Euclidean case in which $\mathrm{cmm}_{4 / 2,4 / 2}=\mathrm{cmm}$. Thus Circle Limit I (Figure 2) has symmetry group $\mathrm{cmm}_{3,2}$. Figure 10 shows a pattern with group $\mathrm{cmm}_{3,3}$.

The symmetry group of the Escher's Circle Limit III pattern (Figure 7) is generated by three rotations: a $90^{\circ}$ rotation about the right fin tip, a $120^{\circ}$ rotation about the left fin tip, and a $120^{\circ}$ rotation about the nose of a fish. The two different kinds of 3 -fold points alternate around the vertices of 8 -gons of the $\{8,3\}$ tessellation. This symmetry group is often denoted $(3,3,4)$. Figure 11 shows a pattern with symmetry group $(3,3,5)$ that is based on the $\{10,3\}$ tessellation. For more on these "Circle Limit III" patterns, see [Dun07b].

\section{Hamiltonian Paths and An Algorithm for Creating Repeating Hyperbolic Patterns}

Given a generating set for a group, the Cayley digraph for that group is the graph whose vertices are group elements and whose edges are labeled by the generators that take one vertex to another (i.e. there is an edge from $u$ to $v$ if there is a generator $g$ such that $v=g u$ ). A Hamiltonian path is a path that meets each group element/vertex exactly once. In the infinite symmetry groups of hyperbolic patterns, we are interested in "one-way" (infinite) paths that start at a vertex (usually the identity) and traverse all the group elements/vertices. The motivation is that by traversing such a path and applying the generator transformations to successive copies of the motif, we can theoretically generate an entire hyperbolic pattern from the original motif. Figure 12 shows a (one-way) Hamiltonian path in the group [6,4]. This is actually an undirected graph since the generators are reflections and reflections are their own inverses.

Where did the path of Figure 12 come from? In the late 1970's I posed the problem of finding such a path to David Witte (Morris). He solved that problem and the related problems of finding Hamiltonian paths in the symmetry groups of all four of Escher's Circle Limit patterns. In fact he, Douglas Jungreis, and I, with help from other undergraduate research students over a few years, published existence and non-existence results for one-way and two-way paths in both directed and undirected Cayley graphs [Dun95]. 


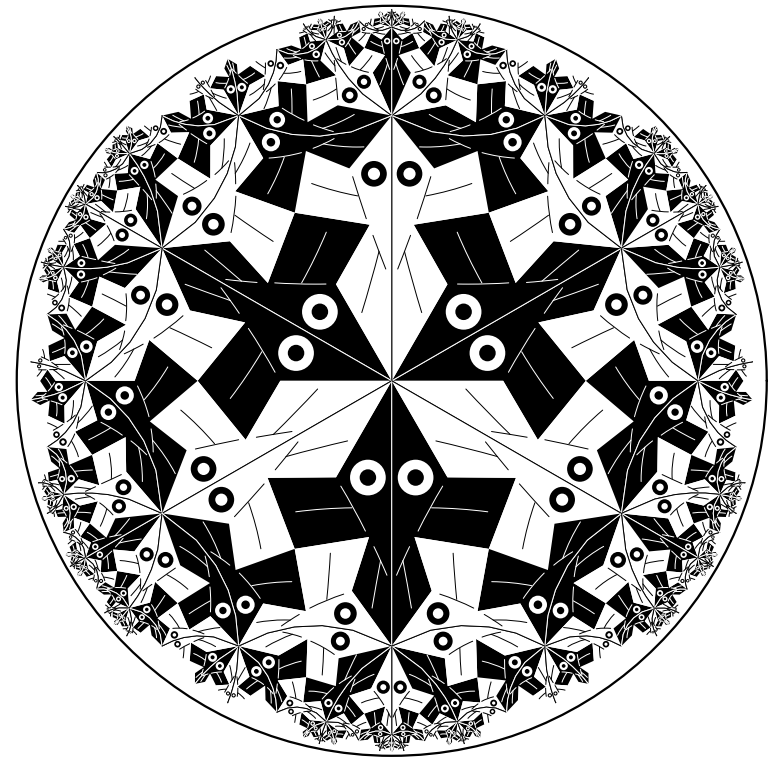

Figure 10: A Circle Limit I fish pattern with symmetry group $\mathrm{cmm}_{3,3}$.

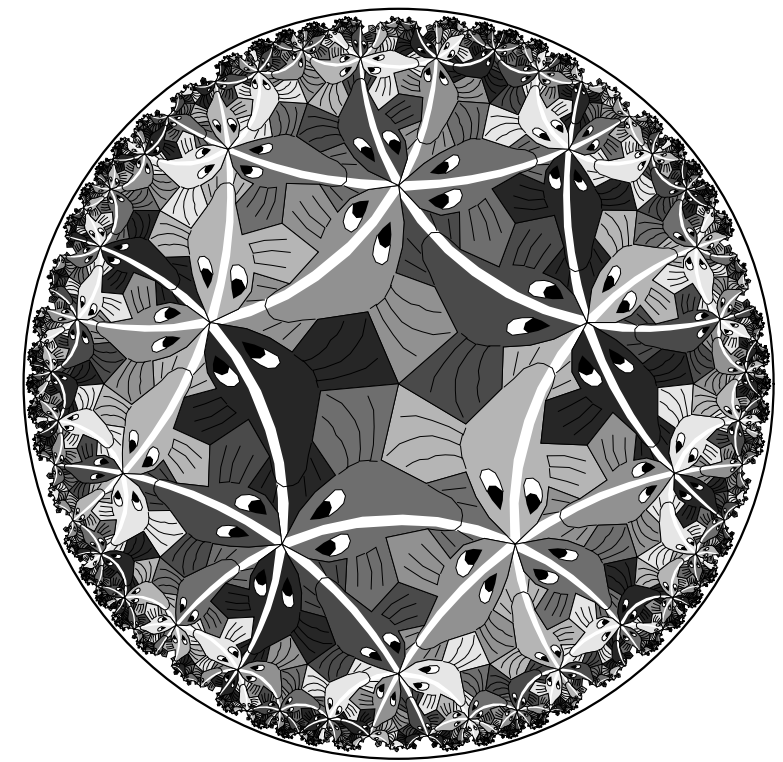

Figure 11: A "Circle Limit III" pattern with symmetry group $(3,3,5)$.

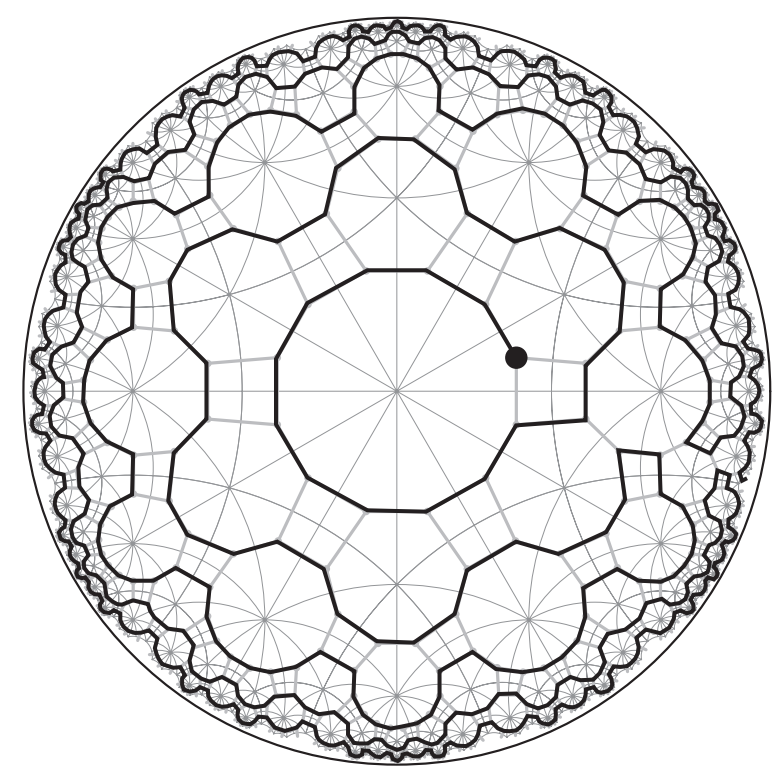

Figure 12: A Hamiltonian path in the group $[6,4]$. 
As suggested above, the motivation for finding Hamiltonian paths was to incorporate them into an algorithm to draw repeating hyperbolic patterns. Specifically, our first goal was to be able to draw each of Escher's four Circle Limit patterns using a computer, thus avoiding the tedious hand-work that Escher had to go through. In examining the path of Figure 12, one notices that it circles around the center most of the time in a single "layer" of triangles, except when it jumps from one layer to the next, and then reverses direction. This was exactly the kind of pattern we wanted for the path, since if we followed it, drawing copies of the motif as we went, we would fill up the Poincaré disk from the center outward without gaps or overlaps. We wanted to avoid gaps to obtain a complete pattern, and we wanted to avoid overlaps not only for efficiency, but because at that time our main hardcopy device was a pen plotter which would tear through the paper if it drew over the same spot too many times. Of course we could only draw a finite number of layers, but this was sufficient to give the idea of the entire infinite pattern. What David Witte Morris came up with were essentially substitution rules for creating the path on the next layer, given the choices on the current layer (there were also the transitions from one layer to the next to figure out).

At this point John Lindgren, a University of Minnesota Duluth undergraduate entered the picture to do the programming. So the setup was: Witte Morris would come up with the rules, I would translate them to pseudocode, and Lindgren would implement it in FORTRAN. The program that we created could generate patterns with several kinds of symmetry groups: $[p, q],[p, q]^{+},\left[p^{+}, q\right], c m m_{3,2}$, and $(3,3, p / 2)$ (I think it may have worked for general $\mathrm{cmm}_{p / 2, q / 2}$ too). Thus we could generate all four of Escher's Circle Limit patterns, which was our goal. We published the results in SIGGRAPH '81 [Dun81]. We generated the figures for that paper using our FORTRAN program, but we also presented a recursive algorithm. Unfortunately there was an error in that algorithm, but it was fixed in a later paper [Dun86].

\section{Other Hyperbolic Pattern-Generating Algorithms}

The first simplification to our algorithm was motivated by the observation that most of Escher's repeating patterns, including all the spherical and hyperbolic ones, were based on regular $\{p, q\}$ tessellations. The idea was to combine all of the motifs within a $p$-gon into a supermotif (which we called a $p$-gon pattern in previous papers). Figure 13 shows the supermotif for Circle Limit I. Then the supermotif could be transformed about the hyperbolic plane. To do this, we found Hamiltonian paths in what we call "Cayley coset graphs" of the desired symmetry groups. The stabilizer, $H$, of the supermotif is used to define cosets of the symmetry group. So the cosets can be represented visually by $p$-gons in the $\{p, q\}$ tessellation, and there is an edge from coset $x H$ to $y H$ if there is a generator $g$ such that $g x H=y H$. These Hamiltonian paths seemed to be easier to find and to program than those for the full group. Figure 14 shows the coset graph edges for the group [6,4] as straight line segments and the 6-gon edges as (light) circular arcs; the Hamiltonian path consists of the dark line segments of the coset graph.

The Hamiltonian path methods led to roundoff errors due to successively multiplying many matrices together to get the current transformation matrix. We actually didn't notice this until we replaced an old but high-precision computer with a newer one with half the precision. This problem was solved by devising a recursive algorithm. The recursive algorithm essentially traversed a spanning tree in the coset graph. Figure 15 shows a spanning tree in the coset graph of the group [6,4]. Again, it was fairly easy to devise spanning trees and to program them. One such algorithm was presented in [Dun86].

A more general recursive algorithm has been devised that removes the restriction that the pattern be based on a $\{p, q\}$ tessellation [Dun07a]. This method assumes that the motif is contained in a finite convex polygon that tiles the hyperbolic plane, and thus has rational angles at its corners. The algorithm proceeds by transforming the motif across sides of copies of that polygon. This algorithm seems more combinatorial than group-theoretic in nature. Figure 16 shows a pattern created by this algorithm — it is based on Escher's "three element" pattern, Notebook Drawing Number 85 (page 184 of [Sch04]). The three elements are a lightly shaded bats for air, a gray lizards for earth, and a dark fish for water (Escher's colors are yellow, red, and blue for the bats, lizards, and fish respectively). 


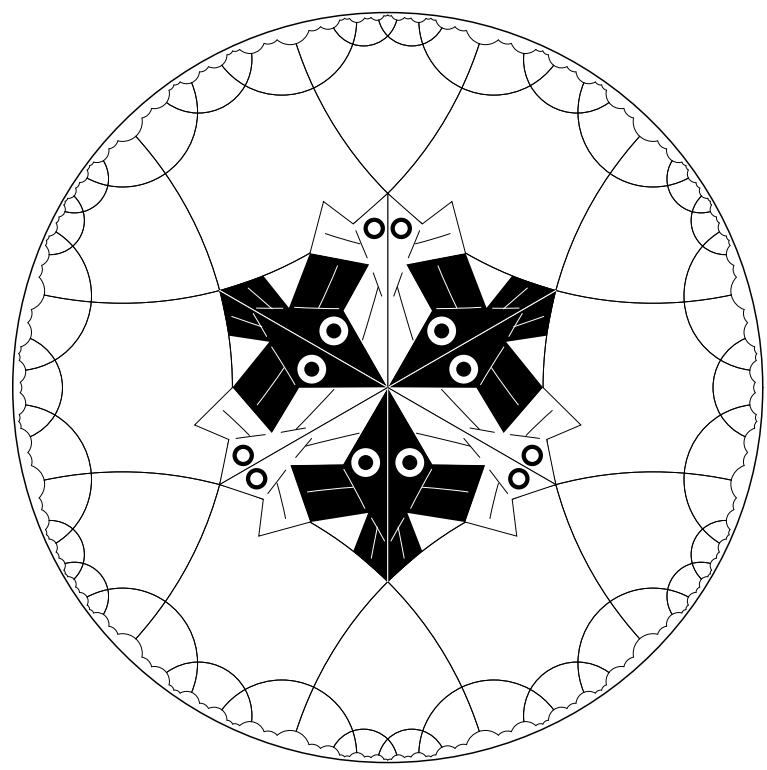

Figure 13: The supermotif for Circle Limit I.

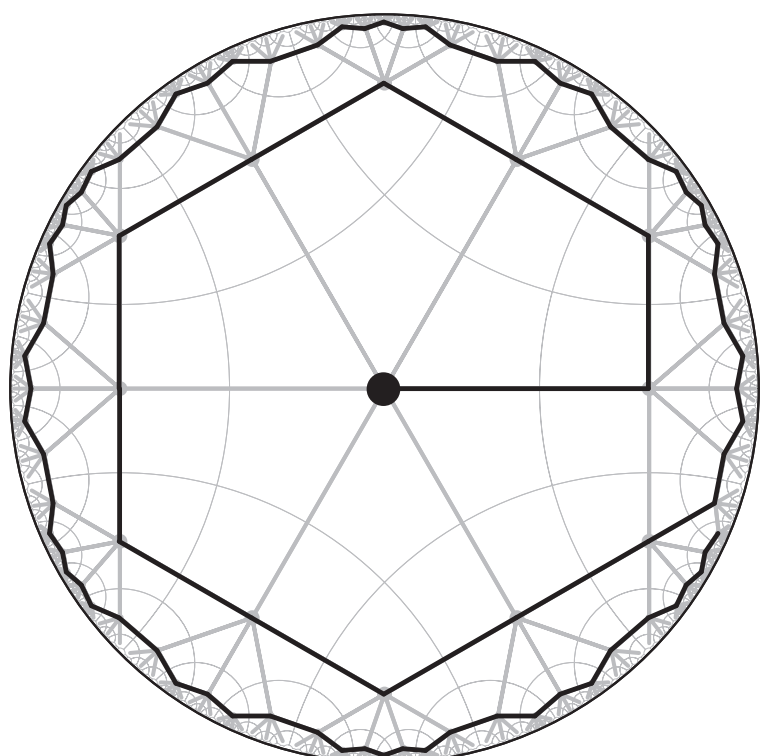

Figure 14: A Hamiltonian path in the coset graph of $[6,4]$.

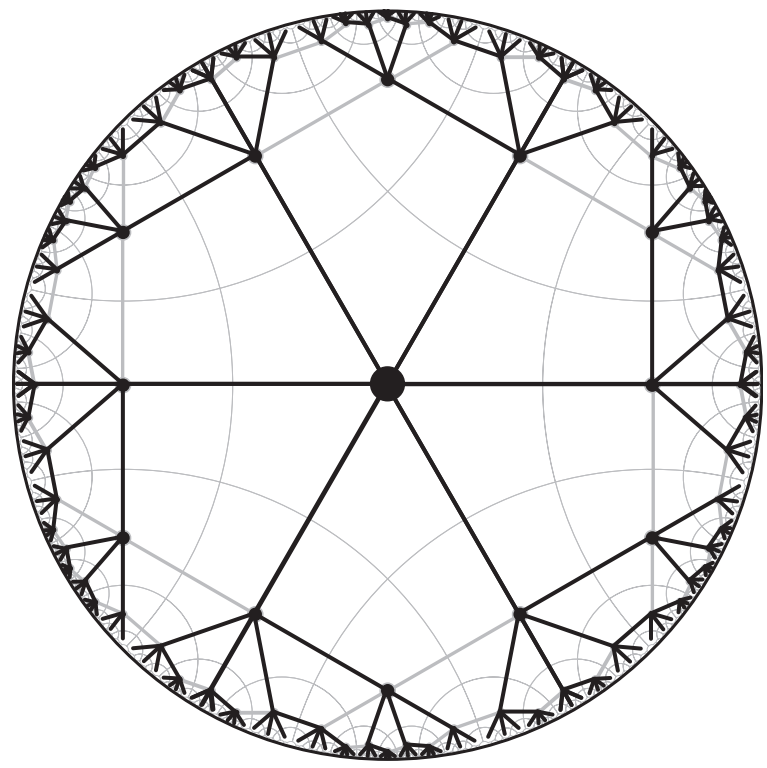

Figure 15: A spanning tree in the coset graph of $[6,4]$.

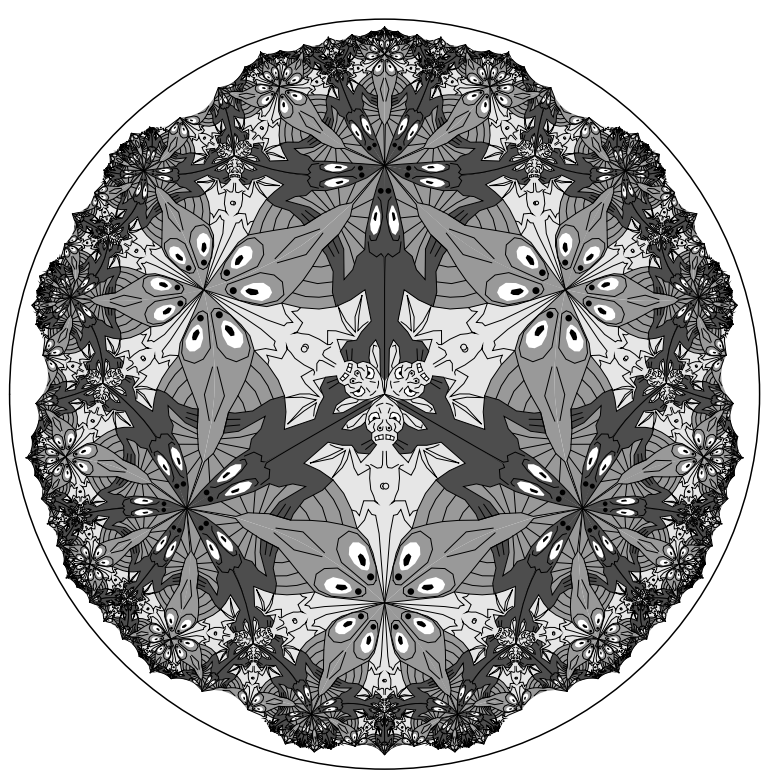

Figure 16: A "three elements" pattern with 3 bats, 5 lizards, and 4 fish meeting at their heads. 


\section{Conclusion and Future Work}

We have shown how Hamiltonian paths were first used to create hyperbolic pattern programs, and then later extended to other algorithms. This was actually a bootstrap process since we used the patterns drawn by one program to figure out an algorithm for the next program. Thus the initial Hamiltonian path program was the key to getting the algorithm development process started.

There are several problems that are yet to be solved. The first is to allow some of the vertices of the motif-enclosing polygon to be on the bounding circle. The second is to transform a motif within one polygon to another polygon. Currently the different versions of the programs can produce patterns with (perfect) color symmetry, but the color permutations must be figured out ahead of time and hand-coded into the motif data files. This leads to the third, and seemingly difficult problem of automating the process of generating patterns with color symmetry, one of the hallmarks of Escher's patterns.

\section{Acknowledgments}

I would like to thank Lisa Fitzpatrick and the staff of the Visualization and Digital Imaging Lab (VDIL) at the University of Minnesota Duluth. This work was also supported by a Summer 2007 VDIL Research grant.

\section{References}

[Cox57] H.S.M. Coxeter, Crystal symmetry and its generalizations, Royal Society of Canada, (3), 51 (1957), pp. 1-13.

[Cox79] H.S.M. Coxeter, The Non-Euclidean Symmetry of Escher's Picture 'Circle Limit III', Leonardo, 12 (1979), pp. 19-25.

[Dun81] D. Dunham, J. Lindgren, D. Witte, Creating repeating hyperbolic patterns, Computer Graphics, Vol. 15, No. 3, August, 1981 (Proceedings of SIGGRAPH '81), pp. 215-223.

[Dun86] D. Dunham, Hyperbolic symmetry, Computers \& Mathematics with Applications (1,2), 12B pp. 139-153, 1986.

[Dun95] D. Dunham, D. Jungreis, David Witte (Morris), Infinite Hamiltonian paths in Cayley digraphs of hyperbolic symmetry groups, Discrete Mathematics 143 (1995), pp. 1-30.

[Dun03] D. Dunham, Hyperbolic Art and the Poster Pattern:

http://www.mathaware.org/mam/03/essay1.html, on the Mathematics Awareness Month 2003 web site: http: //www. mathaware.org/mam/03/.

[Dun07a] D. Dunham, An Algorithm to Generate Repeating Hyperbolic Patterns, in the Proceedings of ISAMA 2007 (eds. Ergun Akleman and Nat Friedman), College Station, Texas, 2007, pp. 111-118.

[Dun07b] D. Dunham, A “Circle Limit III" Calculation, in Bridges Donostia: Mathematical Connections in Art, Music, and Science, (ed. Reza Sarhangi), San Sebastian, Spain, 2007.

[Gre93] M. Greenberg, Euclidean \& Non-Euclidean Geometry, Third Edition: Development and History, 3nd Ed., W. H. Freeman, Inc., New York, 1993. ISBN 0716724464

[Hil01] David Hilbert, Über Flächen von konstanter gausscher Krümmung, Transactions of the American Mathematical Society, pp. 87-99, 1901.

[Sch04] D. Schattschneider, M.C. Escher: Visions of Symmetry, 2nd Ed., Harry N. Abrams, Inc., New York, 2004. ISBN 0-8109-4308-5 